\title{
Diagnostic Value of miR-103 in Patients with Sepsis and Noninfectious SIRS and Its Regulatory Role in LPS-Induced Inflammatory Response by Targeting TLR4
}

\author{
Min Yang $\left(\mathbb{D},{ }^{1}\right.$ Li Zhao $\mathbb{D}^{2},{ }^{2}$ and Mingyan Sun $\mathbb{D D}^{3}$ \\ ${ }^{1}$ Department of Infectious Diseases, Affiliated Hospital of Weifang Medical University, Weifang, Shandong 261031, China \\ ${ }^{2}$ Department of Infectious Diseases, Yidu Central Hospital of Weifang, Weifang, Shandong 262500, China \\ ${ }^{3}$ Clinical Laboratory, Affiliated Hospital of Weifang Medical University, Weifang, Shandong 261031, China
}

Correspondence should be addressed to Mingyan Sun; sunmy_clinic@163.com

Received 15 February 2020; Accepted 28 April 2020; Published 13 May 2020

Academic Editor: Atsushi Kurabayashi

Copyright (c) 2020 Min Yang et al. This is an open access article distributed under the Creative Commons Attribution License, which permits unrestricted use, distribution, and reproduction in any medium, provided the original work is properly cited.

\begin{abstract}
Background. Sepsis is a life-threatening condition and a systemic inflammatory response syndrome (SIRS) driven by infection. This study aimed at investigating the expression of microRNA-103 (miR-103) in sepsis patients, evaluating its diagnostic value, and exploring the regulatory effect of miR-103 on LPS-induced inflammation in monocytes. Methods. Expression of miR-103 was measured using quantitative real-time PCR. A receiver operating characteristics curve was plotted to evaluate the diagnostic vale of miR-103. Serum and cell supernatant levels of proinflammatory cytokines were analyzed using ELISA. The interaction between miR-103 and Toll-like receptors 4 (TLR4) was analyzed using luciferase reporter assay. The effect of miR-103 on inflammation was examined in LPS-treated monocytes. Results. Serum expression of miR-103 was decreased in noninfectious SIRS and sepsis patients compared with healthy controls, and the lowest expression value was observed in sepsis patients (all $P<0.05$ ). Serum levels of miR-103 have considerable diagnostic accuracy in distinguishing sepsis patients from SIRS patients and healthy controls. A negative correlation was found between miR-103 and inflammatory responses in sepsis patients. TLR4 was demonstrated to be a direct target of miR-103 and was negatively regulated by miR-103 in monocytes. The promoted inflammatory responses by LPS in monocytes were reversed by the overexpression of miR-103. Conclusion. All the data revealed that serum decreased miR-103 in sepsis patients serves as a promising noninvasive diagnostic biomarker and may be involved in the pathogenesis of sepsis by regulating inflammatory responses via targeting TLR4.
\end{abstract}

\section{Introduction}

Sepsis is a kind of systemic inflammatory response syndrome (SIRS) driven by infection [1]. It is considered a lifethreatening condition caused by the abnormal host response to infection and a leading cause of deaths in intensive care units (ICUs) [2]. The statistics revealed that there are approximately 250,000 new deaths occurred in ICUs due to sepsis in the United States [3]. The incidence of sepsis is increasing in elderly people, and about $60 \%$ sepsis patients in ICUs are older than 65 years old [4]. Currently, the gold standard for sepsis diagnosis is blood microbiological culture analysis, but this method spends more time to get the examination results than other molecular biomarkers, such as procalcito-
$\operatorname{nin}(\mathrm{PCT})$ and C-reactive protein (CRP) [5]. However, these frequently used molecules lack specificity in distinguishing sepsis cases from noninfectious diseases [6]. Therefore, novel biomarkers with high sensitivity and specificity are necessary for the early diagnosis of sepsis.

MicroRNAs (miRNAs) are a group of small noncoding RNAs with critical regulatory roles in gene expression at posttranscriptional levels [7]. Numerous studies have provided evidence for miRNAs to be involved in the regulation of cellular processes and to serve as promising diagnostic and prognostic biomarkers in various diseases $[8,9]$. A characteristic of miRNAs to be stably detected from serum or plasma samples contributes miRNAs to be a kind of a good diagnostic tool [10]. In patients with sepsis, some miRNAs 
have been identified to be candidate diagnostic biomarkers, such as miR-155-5p and miR-133a-3p [11]. Toll-like receptors (TLRs) have been reported to serve as important roles in sepsis [12]. TLR4 is a widely investigated TLRs in sepsis, which is a key molecule in the innate immune system and the development of sepsis [13]. Some potential therapeutic methods have been demonstrated in sepsis treatment by inhibiting the TLR4 signaling [14]. In this study, we found a complementary sequence of miR-103 at the $3^{\prime}$-untranslated region ( $3^{\prime}$-UTR) of TLR4. Previous studies have demonstrated the regulatory effects of miR-103 on inflammatory responses in some human diseases, such as Alzheimer's disease [15] and atherosclerosis [16]. Of note, a study by Huang et al. investigated the miRNA expression profiles in neonatal sepsis using neonatal monocytes, which are key leukocytes that link innate to adaptive immunity, and found that miR-103 was decreased in LPS-treated cells [17]. The results of the previous studies remind us that miR-103 might also be involved in the development of sepsis.

To improve the diagnosis and treatment of sepsis, this study aimed at investigating the expression of miR-103 in sepsis patients and evaluating the diagnostic potential of miR-103 in distinguishing sepsis patients from noninfectious SIRS patients and healthy individuals. In addition, this study used LPS-treated monocytes to explore the effect of miR-103 on inflammatory responses.

\section{Materials and Methods}

2.1. Patients and Sample Collection. A total of 108 sepsis patients and 89 age- and gender-matched noninfectious SIRS patients were recruited in this study from the ICU of Affiliated Hospital of Weifang Medical University between 2014 and 2017. The diagnosis of patients was performed on the basis of blood microbiological culture results according to the criteria of the American College of Chest Physicians/Society of Critical Care Medicine [18]. The disease severity of the sepsis patients was defined by the sepsis-related organ failure assessment (SOFA) score [19] and the acute physiology and chronic health evaluation (APACHE) II score [20] obtained on day 1 after ICU admission. In addition, 68 healthy volunteers were enrolled in this study as healthy controls, and no statistical significance was presented between the healthy individuals and the sepsis and SIRS patients. Blood samples were collected from the participants at the time of initial diagnosis, and serum was isolated from the blood by centrifugation and stored at $-80^{\circ} \mathrm{C}$. The experimental protocols of this study were approved by the Ethics Committee of Affiliated Hospital of Weifang Medical University, and a written informed consent was obtained from each participant.

2.2. Monocytes Collection and LPS Treatment. The blood samples collected from sepsis patients were settled by $4.5 \%$ dextran 500 (1:5; Amersham Biosciences, Piscataway, NJ, USA), and the leukocytes were separated from the red blood cells. Monocytes were isolated using density gradient centrifugation with Ficoll-Paque (Amersham Pharmacia, Biotech AB, Uppsala, Sweden) as previously described [21]. After the isolation, the monocytes were cultured in RPMI-1640 medium containing $10 \% \mathrm{FBS}$ at $37^{\circ} \mathrm{C}$ in a humidified incubator with $5 \% \mathrm{CO}_{2}$. To simulate the inflammatory response progression of sepsis, $100 \mathrm{ng} / \mathrm{mL}$ lipopolysaccharide (LPS; Sigma-Aldrich, Louis, MO, USA) was used to stimulate monocytes to induce excessive inflammatory responses.

2.3. Cell Transfection. To regulate the expression of miR-103 in monocytes, the cells were transfected with miR-103 mimic, miR-103 inhibitor, or the negative controls (mimic NC and inhibitor NC) (GenePharma, Shanghai, China) by Lipofectamine 2000 (Thermo Fisher Scientific, Waltham, MA, USA) following the manufacturers' protocol. This experiment was repeated three times.

2.4. RNA Extraction and Quantitative Real-Time PCR ( $q R T$ $P C R)$. Total RNA was extracted from monocytes and serum samples collected from 108 sepsis patients, 89 noninfectious SIRS patients, and 68 healthy controls by TRIzol reagent (Invitrogen, Carlsbad, CA, USA), and a NanoDrop 2000 (Thermo Fisher Scientific, Waltham, MA, USA) was used to evaluate the concentration and purity of RNA. The obtained RNA was reversely transcribed into cDNA by a PrimeScript RT reagent kit (TaKaRa, Shiga, Japan) following the manufacturer's instruction. The relative expression of miR103 and mRNA of TLR4 was examined using qRT-PCR, which was conducted by a SYBR green I Master Mix kit (Invitrogen, Carlsbad, CA, USA) and a 7300 Real-Time PCR System (Applied Biosystems, USA). Each sample was examined for at least three times. The final relative expression values were calculated using the $2^{-\Delta \Delta \mathrm{Ct}}$ method and normalized to U6 or GAPDH.

2.5. Enzyme-Linked Immune Sorbent Assay (ELISA). This study measured the serum or cell supernatant levels of proinflammatory cytokines to reflect the inflammatory response status. The levels of IL- $1 \beta$, IL- 6 , and TNF- $\alpha$ were measured using an ELISA kit (Bioscience, San Diego, CA, USA) following the protocols of manufacturers, and the optical density (OD) at $450 \mathrm{~nm}$ was read by a microplate reader (Bio-Rad, Hercules, CA, USA). This experiment was performed in triplicate.

2.6. Luciferase Reporter Assay. This study used miRanda (http://www.microrna.org/microrna/home.do) to predict that TLR4 contains a complementary sequence of miR-103. A subsequent luciferase reporter assay was performed to verify the interaction between miR-103 and TLR4. The wild type (WT) and mutant type (MUT) 3'-UTRs of TLR4 were separately cloned in the pGL3 basic vector (Promega, Madison, $\mathrm{WI}$ ), and the combined vectors were cotransfected into monocytes with miR-103 mimic, miR-103 inhibitor, or the NCs using Lipofectamine 2000 (Thermo Fisher Scientific, Waltham, MA, USA). The luciferase activity in each group was measured using a Dual-Luciferase Reporter Assay System (Promega), and this assay was repeated three times.

2.7. Statistical Analysis. The data obtained from this study was expressed as mean \pm SD and analyzed using the SPSS 18.0 software (SPSS Inc., Chicago, IL) and GraphPad Prism 


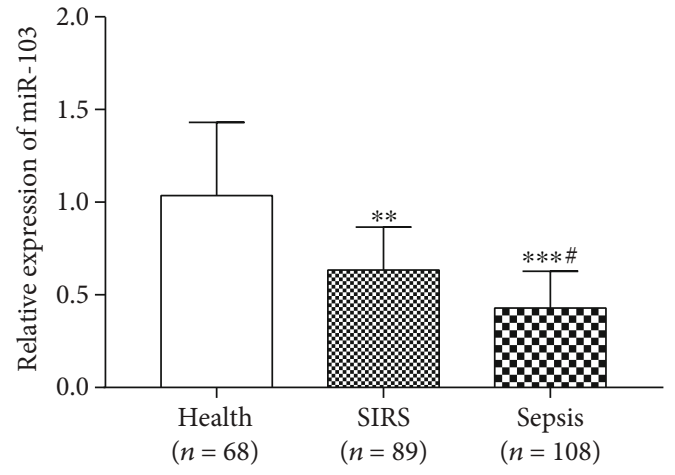

Figure 1: Expression of serum miR-103 in sepsis patients $(n=108)$, noninfectious SIRS patients $(n=89)$, and healthy controls $(n=68)$. ${ }^{* *} P<0.01$ and ${ }^{* * *} P<0.001$ compared with the healthy group; ${ }^{\#} P<0.05$ compared with the SIRS group.

5.0 software (GraphPad Software, Inc., USA). Differences between groups were compared using Student's $t$ test, oneway ANOVA, or Chi-squared test. Correlations between indicators are assessed using Pearson's correlation coefficient. A receiver operating characteristics (ROC) curve was performed to evaluate the diagnostic performance of miR103. All the analyses were repeated at least three times. A difference with a $P<0.05$ indicated statistically significant.

\section{Results}

3.1. Serum Expression of miR-103 in Sepsis Patients. According to qRT-PCR, the serum expression of miR-103 was examined. As shown in Figure 1, the expression of miR-103 was decreased in both the noninfectious SIRS patients $(n=89)$ and sepsis patients $(n=108)$ compared with the healthy controls $(n=68)$ (both $P<0.01)$. In addition, a lower expression of miR-103 was observed in sepsis patients when compared to the expression results in the noninfectious SIRS patients $(P<0.05)$.

3.2. Correlation between $m i R-103$ and Clinicopathological Characteristics of Patients with Sepsis. This study summarized the clinicopathological features of the sepsis patients, including body mass index (BMI), white blood cell (WBC), CRP, PCT, APACHE II score, and SOFA score. To analyze the potential role of miR-103 in sepsis development, the correlation between miR-103 and the clinical data of sepsis patients was assessed. In Table 1, we found that the expression of miR-103 was negatively correlated with the WBC, CRP, PCT, APACHE II score, and SOFA score of sepsis patients (all $P<0.001$ ), while there was no significant correlation between miR-103 and BMI $(P>0.05)$.

3.3. Diagnostic Performance of Serum Levels of miR-103. Given the deregulated expression of miR-103 in SIRS patients and sepsis patients compared with healthy individuals, the diagnostic potential of miR-103 was further evaluated. As shown in Figure 2(a), the area under the curve (AUC) was 0.830 for miR-103 to distinguish SIRS patients from healthy controls. The results shown in Figure 2(b)
TABLE 1: Correlation of miR-103 with clinical characteristics of 108 sepsis patients.

\begin{tabular}{lcc}
\hline Indicators & \multicolumn{1}{c}{ miR-103 } \\
& Correlation coefficient $(r)$ & $P$ value \\
\hline BMI & 0.181 & 0.061 \\
WBC & -0.818 & $<0.001$ \\
CRP & -0.736 & $<0.001$ \\
PCT & -0.674 & $<0.001$ \\
APACHE II score & -0.767 & $<0.001$ \\
SOFA score & -0.680 & $<0.001$ \\
\hline
\end{tabular}

BMI: body mass index; WBC: white blood cell; CRP: C-reactive protein; PCT: procalcitonin; APACHE: acute physiology and chronic health evaluation; SOFA: sepsis-related organ failure assessment.

revealed the high diagnostic accuracy of miR-103 in the distinguishing between sepsis patients from healthy individuals, as evidenced by the AUC of 0.916 and sensitivity and specificity of $89.8 \%$ and $88.2 \%$ at a cutoff value of 0.685 . Moreover, the diagnostic performance of $\mathrm{miR}-103$ in the differentiation of sepsis from SIRS was also examined. As shown in Figure 2(c), the AUC was 0.783 with a sensitivity and specificity of $83.3 \%$ and $76.4 \%$ at a cutoff value of 0.525 , indicating the diagnostic value of miR-103 in the screening of sepsis patients from noninfectious SIRS patients.

3.4. Correlation of miR-103 with Inflammation of Sepsis Patients. This study analyzed the correlation of miR103 with inflammatory responses in sepsis patients. The results listed in Table 2 indicated that serum levels of miR-103 were negatively correlated with the levels of IL- $\beta(r=-0.691, P<$ $0.001)$, IL-6 $(r=-0.725, P<0.001)$, and TNF- $\alpha(r=-0.654$, $P<0.001)$ in patients with sepsis.

3.5. Expression of miR-103 and TLR4 in LPS-Treated Monocytes. The collected monocytes were treated with LPS to simulate the pathogenesis of sepsis. The expression of miR-103 and TLR4 was measured by qRT-PCR in monocytes stimulated by LPS. The results shown in Figures 3(a) and 3(b) indicated that the mRNA expression of TLR 4 was as expected to be increased in the LPS-treated group, while the relative expression of miR-103 was decreased in monocytes treated with LPS (both $P<0.001$ ). A complementary sequence of miR-103 was predicted at the $3^{\prime}$-UTR of TLR4 (Figure 3(c)), and the subsequent luciferase reporter assay was conducted to confirm their interaction. As shown in Figure $3(\mathrm{~d})$, the relative luciferase activity was significantly inhibited by the overexpression of miR-103 but was promoted by the reduction of miR-103 in the WT group (both $P<0.05)$. No significant changes were found in the luciferase activity in MUT group $(P>0.05)$. Furthermore, the regulatory effect of miR-103 on the expression of TLR4 was checked by regulating the expression of miR-103 in monocytes. The cell transfection efficiency results showed that the expression of miR-103 was successfully upregulated by the miR-103 mimic and was downregulated by the miR-103 inhibitor (both $P<0.001$, Figure 3(e)). The mRNA expression of TLR4 results shown in Figure 3(f) revealed that the 


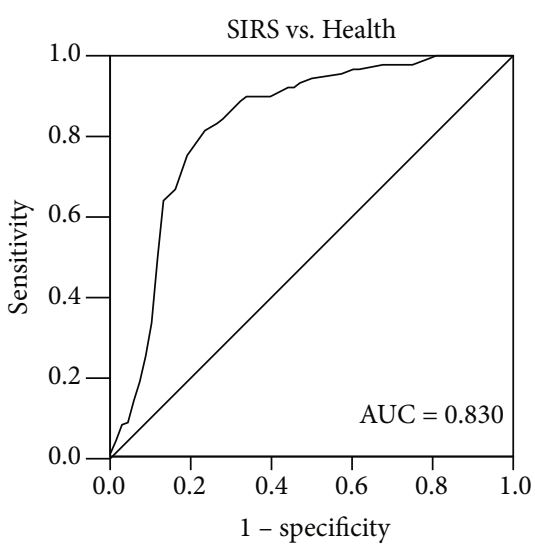

(a)

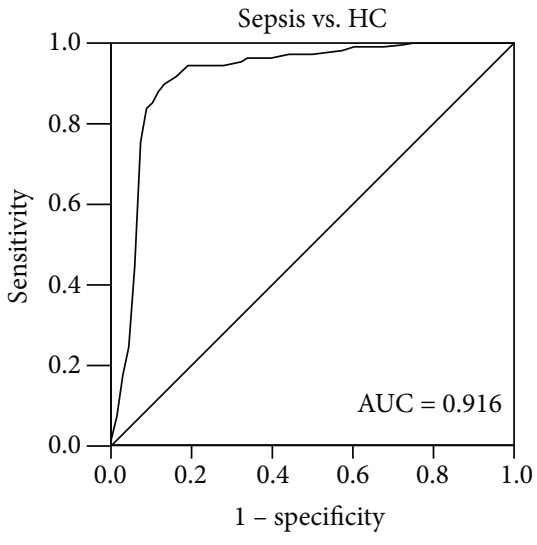

(b)

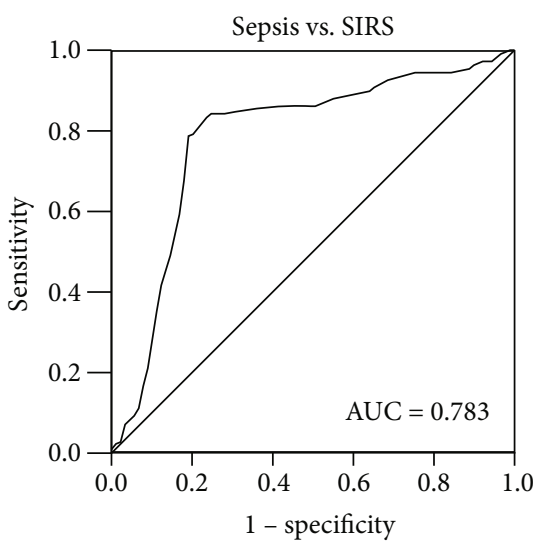

(c)

FIGURE 2: ROCs for SIRS and sepsis patients based on serum miR-103 expression levels. (a) A ROC for the differentiation between noninfectious SIRS patients $(n=89)$ from healthy individuals $(n=68)$. (b) A ROC in distinguishing sepsis patients $(n=108)$ from healthy individuals $(n=68)$. (c) A ROC for the differentiation between sepsis cases $(n=108)$ from noninfectious SIRS $(n=89)$. AUC: area under the curve.

TABLE 2: Correlation of miR-103 with proinflammatory cytokines in 108 sepsis patients.

\begin{tabular}{lcc}
\hline Indicators & \multicolumn{1}{c}{$\operatorname{miR}-103$} & \\
& Correlation coefficient $(r)$ & $P$ value \\
\hline IL-1 $\beta$ & -0.691 & $<0.001$ \\
IL-6 & -0.725 & $<0.001$ \\
TNF- $\alpha$ & -0.654 & $<0.001$ \\
\hline
\end{tabular}

IL: interleukin; TNF: tumor necrosis factor.

upregulation of miR-103 could inhibit, while the downregulation of miR-103 could promote, TLR4 expression in the LPS-treated monocytes (all $P<0.01$ ).

3.6. Effect of miR-103 on Inflammatory Response in LPSTreated Monocytes. After the in vitro manipulation of miR103 , the changes in inflammatory responses in monocytes treated by LPS were further investigated. As shown in Figure 4 , we observed that the increased levels of IL- $1 \beta$, IL6 , and TNF- $\alpha$ induced by LPS stimulation were all reversed by the overexpression of miR-103, while aggravated by the knockdown of miR-103 in monocytes (all $P<0.01$ ).

\section{Discussion}

Dysregulation of miRNAs have attracting increasing attention for their critical roles in the pathogenesis of various human diseases, including inflammatory diseases such as SIRS [22]. Sepsis is considered a SIRS caused by infection, and the understanding about the immunopathology of SIRS and sepsis is important for the development of novel diagnostic and therapeutic approaches [23]. Currently, some aberrantly expressed miRNAs have been identified in sepsis. For example, the decreased miR-23b expression detected in the peripheral blood mononuclear cells of sepsis patients was negatively correlated with inflammatory response and could alleviate the LPS-stimulated inflammatory cytokines [24]. The decreased expression of miR-21 was reported in patients with sepsis and exhibited a good value to predict sepsis risk and thus was determined as a candidate biomarker for the development and progression of sepsis [25]. In patients collected from ICU, a low-level serum of miR-143 was indicated to be an indicator to predict the onset of sepsis and disease severity [26]. These previous results indicate the critical roles of deregulated miRNAs in the pathogenesis of sepsis. 


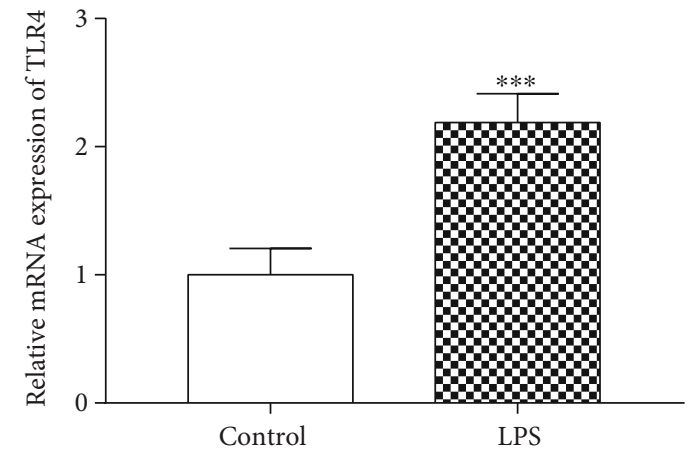

(a)

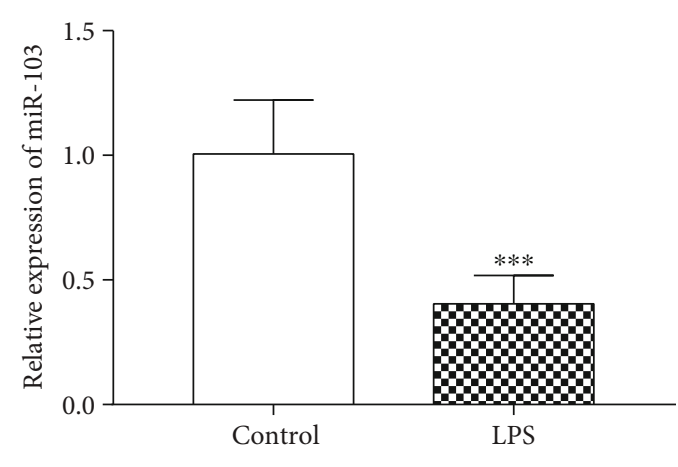

(b)
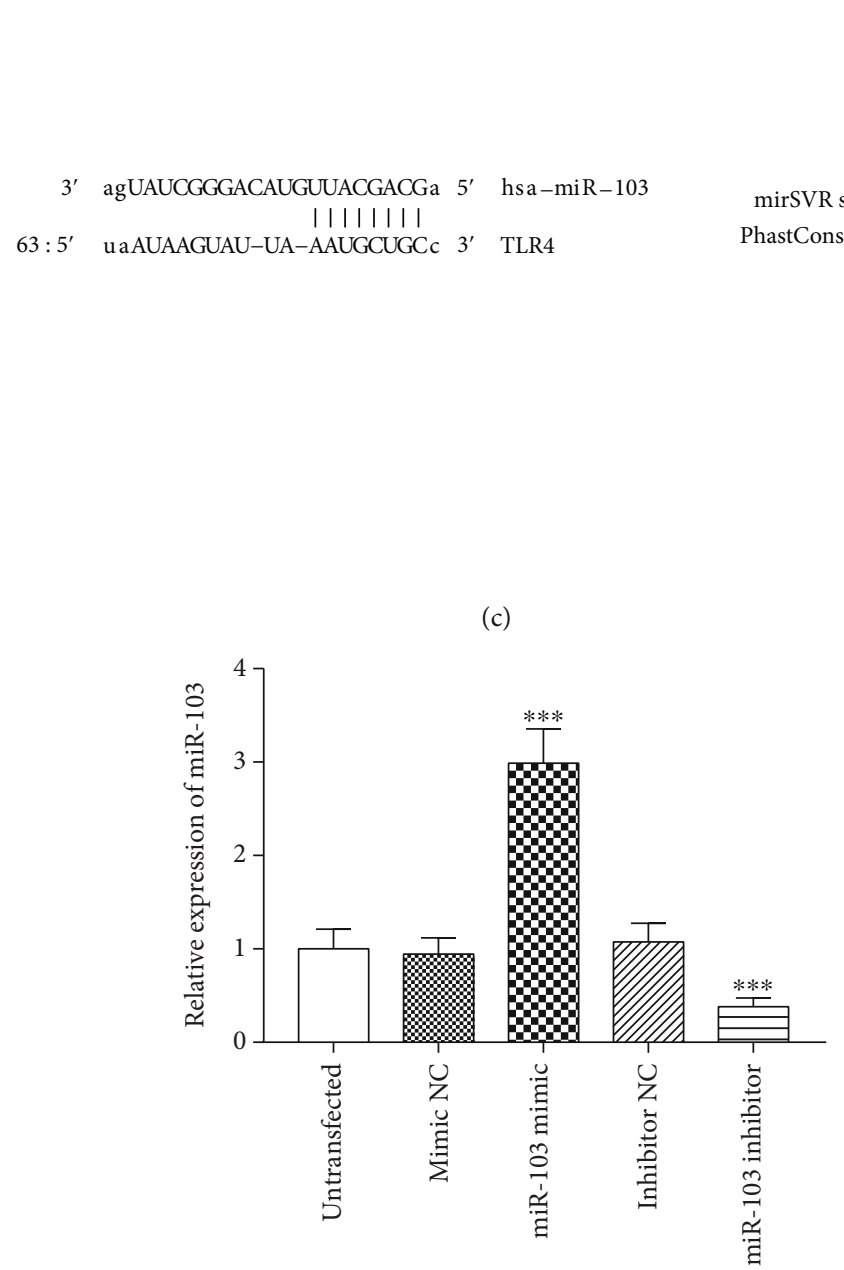

(e)

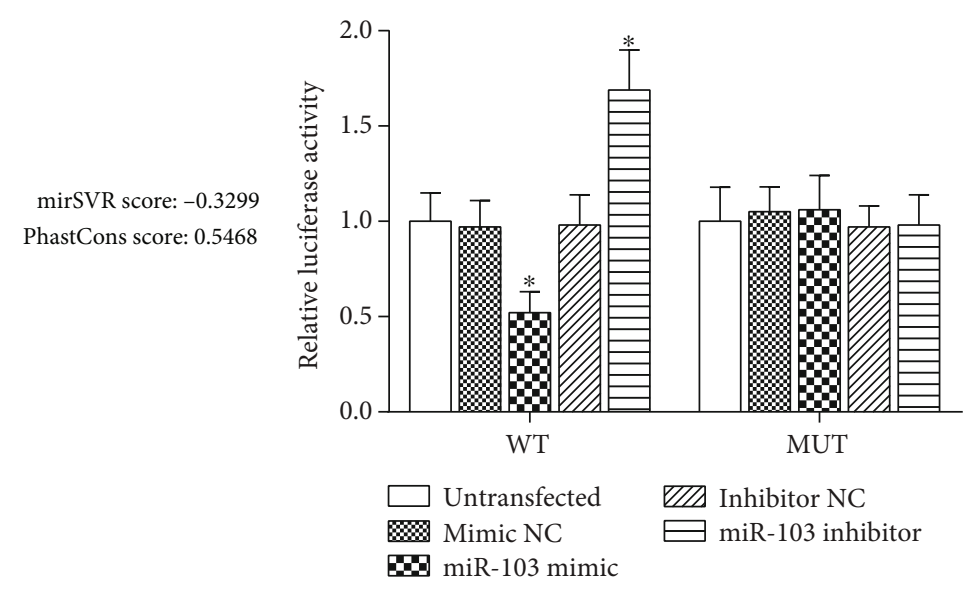

(d)

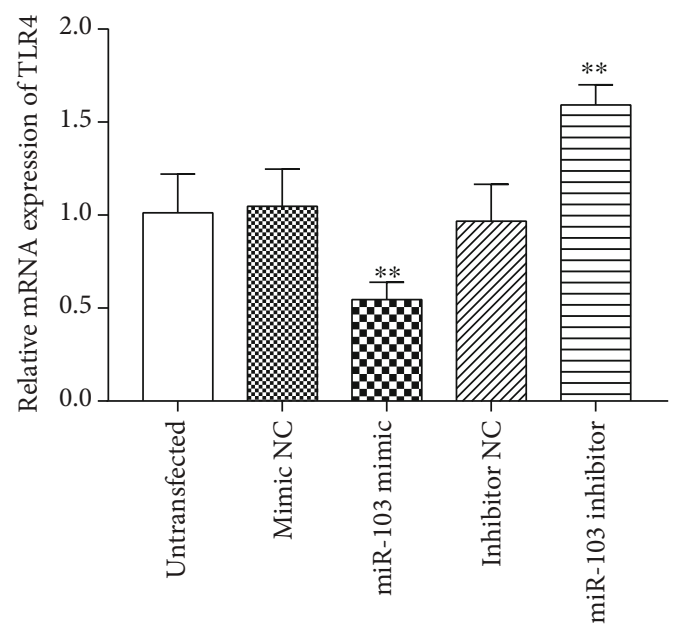

(f)

FIGURE 3: miR-103 directly inhibited the expression of TLR4 in monocytes under LPS treatment. (a, b) Relative expression of TLR3 mRNA (a) and miR-103 (b) in monocytes treated with LPS $\left({ }^{* * *} P<0.001\right)$. (c) The predictive binding site of miR-103 on the $3^{\prime}$-UTR of TLR4. (d) Luciferase activity results to confirm the interaction of miR-103 and TLR4 ( ${ }^{*} P<0.05$ compared with the untransfected group). (e) Cell transfection efficiency examination results $\left({ }^{* * *} P<0.001\right.$ compared with the untransfected group). (f) Inhibiting effect of miR-103 on the mRNA expression of TLR4 in LPS-treated monocytes $\left({ }^{* *} P<0.01\right.$ compared with the untransfected group). The data were collected from the experiments that were repeated at least three times.

A study by Huang et al. reported the dysregulation of miR-103 in neonatal sepsis, and that miR-103 expression was reduced in neonatal monocytes treated by LPS [17]. In this study, we first investigated the expression of miR-103 in adult sepsis and found that serum expression of miR-103 was decreased in noninfectious SIRS and sepsis patients compared with healthy individuals, and that a lower miR-103 level was demonstrated in sepsis than that in the noninfectious SIRS. In addition, the significant association between miR-103 and the clinical characteristics, including WBC, 


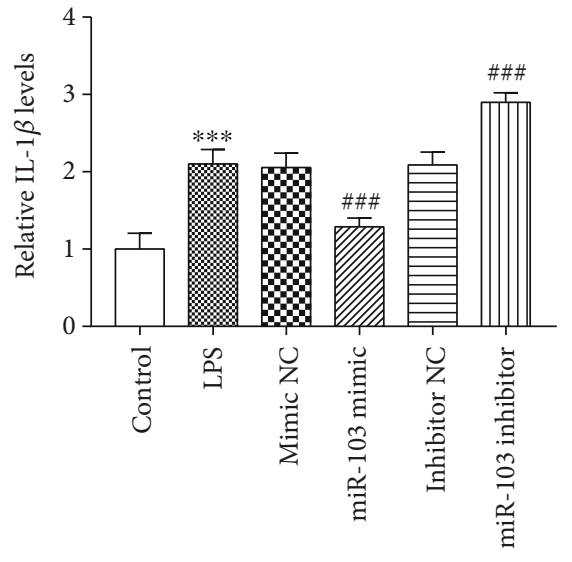

(a)

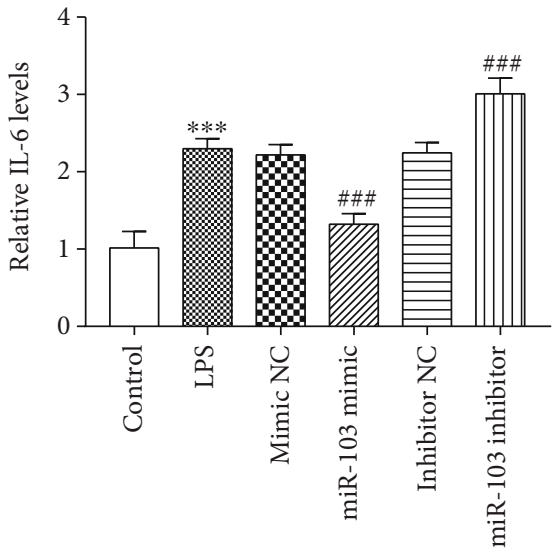

(b)

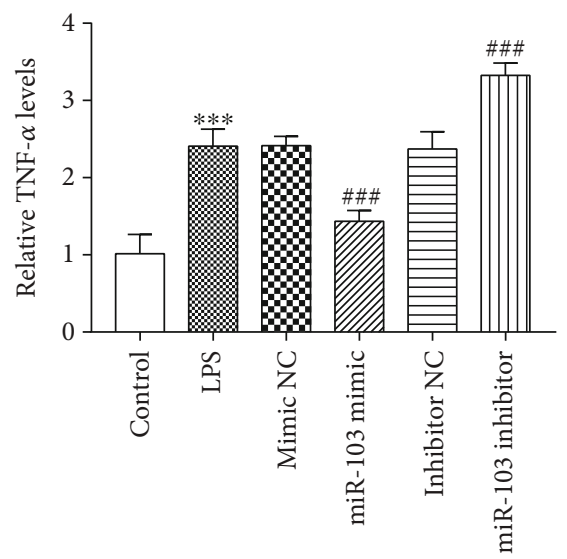

(c)

FIGURE 4: Effect of miR-103 on inflammation in LPS-treated monocytes. The levels of IL-1 $\beta$ (a), IL-6 (b), and TNF- $\alpha$ (c) in cell culture supernatants were inhibited by the overexpression but were enhanced by the knockdown of miR-103. The data were collected from the experiments that were repeated at least three times. ${ }^{* * *} P<0.001$ compared with the control group; ${ }^{\# \# \# ~} P<0.001$ compared with the LPS group.

CRP, PCT, APACHE II score, and SOFA score, was found in sepsis patients, implying that miR-103 might be involved in the development and progression of sepsis.

miRNAs can be easily detected from serum and plasma samples, making them a group of good diagnostic tools in various diseases [27]. Guo et al. provided evidence for the decreased serum miR-495 as a diagnostic biomarker to screen sepsis patients from healthy population [28]. Lan et al. reported that serum miR-155-5p and miR-133a-3p levels were increased in sepsis and related with disease severity, which served as candidate biomarkers for the diagnosis of sepsis [11]. The downregulated miR-146a expression in sepsis patients was determined to be a diagnostic biomarker in distinguishing sepsis patients from non-sepsis-SIRS patients [6]. Considering the lowest miR-103 expression in sepsis patients compared with noninfectious SIRS patients and healthy volunteers, the diagnostic potential of miR-103 was further evaluated. The ROC analysis data indicated that the decreased expression of miR-103 had relatively high diagnostic accuracy in differentiation of sepsis and noninfectious SIRS from healthy individuals. It is worth noting that the low expression of miR-103 also had considerable diagnostic value for distinguishing sepsis patients from noninfectious
SIRS patients. Since sepsis is a life-threatening condition, early diagnosis, especially the screening of sepsis from nonsepsis-SIRS, is of great importance for timely treatment. Our results provided evidence for miR-103 to serve as a candidate biomarker to screen sepsis cases from SIRS patients.

Previous studies have showed that miR-103 plays an important regulatory role in inflammatory responses, such as the inhibiting effect of miR-103 on inflammation in obesity and metabolic syndrome-related disorders [29], chronic obstructive pulmonary disease [30], and Alzheimer's disease [15]. In the present study, the serum miR-103 was found to be negatively correlated with inflammatory cytokine levels in sepsis patients. Moreover, the overexpression of miR-103 could inhibit the LPS-induced inflammatory response in monocytes. These results suggested that miR-103 might be a potential therapeutic target of sepsis by ameliorating the pathologic inflammation. TLR4 is one of the widely studied TLRs, which acts as a key molecule in the regulation of inflammatory response in sepsis [13]. The $3^{\prime}$-UTR of TLR4 contained a complementary sequence of miR-103, and we demonstrated that miR-103 could directly bind to the $3^{\prime}$ -UTR of TLR4 and negatively regulated TLR4 mRNA expression in monocytes. The important role of TLR4 in sepsis 
combined with our study results led us to deduce that miR103 might inhibit the inflammatory response of sepsis by targeting TLR4.

In conclusion, this study demonstrated that serum having decreased miR-103 serves as a candidate diagnostic biomarker to screen sepsis patients from noninfectious SIRS patients and healthy population. The overexpression of miR-103 may be a promising therapeutic target of sepsis by attenuating inflammatory response through the TLR4 signaling. Although this study provides a novel insight into the diagnosis and therapy of sepsis, the clinical significance of serum miR-103 and the functional role of miR-103 in sepsis are needed to be confirmed in further studies.

\section{Data Availability}

All data generated or analyzed during this study are included in this published article.

\section{Ethical Approval}

The experimental protocols of this study were approved by the Ethics Committee of Affiliated Hospital of Weifang Medical University, and a written informed consent was obtained from each participant.

\section{Conflicts of Interest}

The authors declare that they have no competing interests.

\section{Authors' Contributions}

MY and MS designed this study, conducted the clinical research, and wrote the manuscript. LZ performed the cell experiments and analyzed the cell data.

\section{References}

[1] J. Rello, F. Valenzuela-Sanchez, M. Ruiz-Rodriguez, and S. Moyano, "Sepsis: a review of advances in management," Advances in Therapy, vol. 34, no. 11, pp. 2393-2411, 2017.

[2] R. Postelnicu and L. Evans, "Monitoring of the physical exam in sepsis," Current Opinion in Critical Care, vol. 23, no. 3, pp. 232-236, 2017.

[3] R. S. Hotchkiss, G. Monneret, and D. Payen, "Sepsis-induced immunosuppression: from cellular dysfunctions to immunotherapy," Nature Reviews Immunology, vol. 13, no. 12, pp. 862-874, 2013.

[4] R. S. Hotchkiss, G. Monneret, and D. Payen, "Immunosuppression in sepsis: a novel understanding of the disorder and a new therapeutic approach," The Lancet Infectious Diseases, vol. 13, no. 3, pp. 260-268, 2013.

[5] M. Sandquist and H. R. Wong, "Biomarkers of sepsis and their potential value in diagnosis, prognosis and treatment," Expert Review of Clinical Immunology, vol. 10, no. 10, pp. 1349-1356, 2014.

[6] L. Wang, H. C. Wang, C. Chen et al., "Differential expression of plasma miR-146a in sepsis patients compared with nonsepsis-SIRS patients," Experimental and Therapeutic Medicine, vol. 5, no. 4, pp. 1101-1104, 2013.
[7] Z. Li and T. M. Rana, "Therapeutic targeting of microRNAs: current status and future challenges," Nature Reviews Drug Discovery, vol. 13, no. 8, pp. 622-638, 2014.

[8] N. Coban, D. Pirim, A. F. Erkan, B. Dogan, and B. Ekici, "HsamiR-584-5p as a novel candidate biomarker in Turkish men with severe coronary artery disease," Molecular Biology Reports, vol. 47, no. 2, pp. 1361-1369, 2020.

[9] C. Chu, X. Lei, Y. Li et al., "High expression of miR-222-3p in children with mycoplasma pneumoniae pneumonia," Italian Journal of Pediatrics, vol. 45, no. 1, p. 163, 2019.

[10] T. Tian, J. Wang, and X. Zhou, "A review: microRNA detection methods," Organic \& Biomolecular Chemistry, vol. 13, no. 8, pp. 2226-2238, 2015.

[11] C. Lan, X. Shi, N. Guo, H. Pei, and H. Zhang, "Value of serum miR-155-5p and miR-133a-3p expression for the diagnosis and prognosis evaluation of sepsis," Zhonghua Wei Zhong Bing Ji Jiu Yi Xue, vol. 28, no. 8, pp. 694-698, 2016.

[12] H. R. Li, J. Liu, S. L. Zhang et al., "Corilagin ameliorates the extreme inflammatory status in sepsis through TLR4 signaling pathways," BMC Complementary and Alternative Medicine, vol. 17, no. 1, p. 18, 2017.

[13] N. Chantratita, S. Tandhavanant, S. Seal et al., "TLR4 genetic variation is associated with inflammatory responses in Grampositive sepsis," Clinical Microbiology and Infection, vol. 23, no. 1, pp. 47.e1-47.e10, 2017.

[14] L. Loubaki, D. Chabot, I. Pare, M. Drouin, and R. Bazin, "MiR146a potentially promotes IVIg-mediated inhibition of TLR4 signaling in LPS-activated human monocytes," Immunology Letters, vol. 185, pp. 64-73, 2017.

[15] H. Yang, H. Wang, H. Shang et al., "Circular RNA circ_ 0000950 promotes neuron apoptosis, suppresses neurite outgrowth and elevates inflammatory cytokines levels via directly sponging miR-103 in Alzheimer's disease," Cell Cycle, vol. 18, no. 18, pp. 2197-2214, 2019.

[16] P. Hartmann, Z. Zhou, L. Natarelli et al., "Endothelial Dicer promotes atherosclerosis and vascular inflammation by miRNA-103-mediated suppression of KLF4," Nature Communications, vol. 7, no. 1, article 10521, 2016.

[17] H. C. Huang, H. R. Yu, L. T. Huang et al., "miRNA-125b regulates TNF- $\alpha$ production in $\mathrm{CD}_{1} 4^{+}$neonatal monocytes via post-transcriptional regulation," Journal of Leukocyte Biology, vol. 92, no. 1, pp. 171-182, 2012.

[18] R. C. Bone, R. A. Balk, F. B. Cerra et al., "Definitions for sepsis and organ failure and guidelines for the use of innovative therapies in sepsis. The ACCP/SCCM Consensus Conference Committee. American College of Chest Physicians/Society of Critical Care Medicine," Chest, vol. 101, no. 6, pp. 16441655, 1992.

[19] J. -L. Vincent, R. Moreno, J. Takala et al., "The SOFA (Sepsisrelated Organ Failure Assessment) score to describe organ dysfunction/failure. On behalf of the Working Group on SepsisRelated Problems of the European Society of Intensive Care Medicine," Intensive Care Medicine, vol. 22, no. 7, pp. 707710, 1996.

[20] W. A. Knaus, E. A. Draper, D. P. Wagner, and J. E. Zimmerman, "APACHE II: a severity of disease classification system," Critical Care Medicine, vol. 13, no. 10, pp. 818-829, 1985.

[21] H. R. Yu, R. F. Chen, K. C. Hong et al., "IL-12-independent Th1 polarization in human mononuclear cells infected with varicella-zoster virus," European Journal of Immunology, vol. 35, no. 12, pp. 3664-3672, 2005. 
[22] S. Caserta, F. Kern, J. Cohen, S. Drage, S. F. Newbury, and M. J. Llewelyn, "Circulating plasma microRNAs can differentiate human sepsis and systemic inflammatory response syndrome (SIRS)," Scientific Reports, vol. 6, no. 1, article 28006, 2016.

[23] N. A. Rahman, C. M. Chan, M. I. Zakaria, and M. J. Jaafar, "Knowledge and attitude towards identification of systemic inflammatory response syndrome (SIRS) and sepsis among emergency personnel in tertiary teaching hospital," Australasian Emergency Care, vol. 22, no. 1, pp. 13-21, 2019.

[24] W. Zhang, F. Lu, Y. Xie et al., "miR-23b negatively regulates sepsis-induced inflammatory responses by targeting ADAM10 in human THP-1 monocytes," Mediators of Inflammation, vol. 2019, Article ID 5306541, 13 pages, 2019.

[25] L. Na, H. Ding, E. Xing et al., "The predictive value of microRNA-21 for sepsis risk and its correlation with disease severity, systemic inflammation, and 28-day mortality in sepsis patients," Journal of Clinical Laboratory Analysis, vol. 34, no. 3, article e23103, 2020.

[26] C. Roderburg, A. Koch, F. Benz et al., "Serum levels of miR-143 predict survival in critically ill patients," Disease Markers, vol. 2019, Article ID 4850472, 10 pages, 2019.

[27] R. Hamam, D. Hamam, K. A. Alsaleh et al., "Circulating microRNAs in breast cancer: novel diagnostic and prognostic biomarkers," Cell Death \& Disease, vol. 8, no. 9, article e3045, 2017.

[28] H. Guo, L. Tang, J. Xu et al., "MicroRNA-495 serves as a diagnostic biomarker in patients with sepsis and regulates sepsisinduced inflammation and cardiac dysfunction," European Journal of Medical Research, vol. 24, no. 1, p. 37, 2019.

[29] Z. Zhang, S. Wu, S. Muhammad, Q. Ren, and C. Sun, "miR103/107 promote ER stress-mediated apoptosis via targeting the Wnt3a/beta-catenin/ATF6 pathway in preadipocytes," Journal of Lipid Research, vol. 59, no. 5, pp. 843-853, 2018.

[30] Z. Xi, Y. Qiao, J. Wang et al., "Gastrodin relieves inflammation injury induced by lipopolysaccharides in MRC- 5 cells by upregulation of miR-103," Journal of Cellular and Molecular Medicine, vol. 24, no. 2, pp. 1451-1459, 2020. 